\title{
EL ANÁLISIS DE IMPACTO PRESUPUESTARIO EN SALUD: PUESTA AL DÍA CON UN MODELO DE ABORDAJE GENÉRICO
}

\author{
Osvaldo Ulises Garay,a, Joaquín E. Caporale ${ }^{1, b}$, Andrés Pichón-Riviere ${ }^{1, c}$, Sebastián García Martí1,d, \\ Mercedes Mac Mullen ${ }^{1, a}$, Federico Augustovski ${ }^{1, e}$
}

\begin{abstract}
RESUMEN
El Análisis de Impacto Presupuestario (AIP) en el campo de la salud puede ser definido como la estimación de los costos financieros netos que le representarían a una institución dar cobertura a una determinada intervención. En la práctica, los AIP se utilizan frecuentemente para decidir la inclusión o exclusión de medicamentos en formularios terapéuticos y notoriamente han obligado a reconocer que las Evaluaciones Económicas representan una mirada parcial en el análisis de las consecuencias de la incorporación de tecnologías sanitarias. Este trabajo procura identificar los determinantes y componentes de los análisis de impacto presupuestario, y a partir de ello describir el desarrollo de un modelo creado en una planilla de cálculo que permite considerar cualquier tecnología sanitaria y obtener estimaciones con diferentes grados de complejidad. Su diseño incorpora de forma explícita las habilidades del usuario y las deficiencias de información, buscando a su vez promover el desarrollo de estas herramientas en los ámbitos de gestión de nuestros países.
\end{abstract}

Palabras clave: Economía de la salud; Costos de atención médica; Costos y análisis de costo; Economía Farmacéutica; metodología (fuente: DeCS BIREME).

\section{BUDGETARY IMPACT ANALYSIS IN HEALTH: UPDATE WITH A MODEL USING A GENERIC APPROACH}

\begin{abstract}
Budgetary Impact Analysis (BIA) applied to health care can be defined as the estimate of the net financial costs that a given intervention would represent for a health care institution given the case it was covered. Routinely, BIAs are used to decide the inclusion or exclusion of drugs in therapeutic schemes; actually, the increased use of BIAs have raised awareness about the fact that health economic evaluations represent a partial view in the analysis of the consequences of incorporating health technologies. This paper seeks to identify the determinants and components of BIA, and to describe the development of a spreadsheet model that enables us to assess the Budget impact of any health technology and perform estimations with differing degrees of complexity. Its design explicitly adapts to the user skills and gaps in information, thus seeking to promote the development of these tools in the management fields in our countries.
\end{abstract}

Key words: Health economics; Health care costs; Costs and cost analysis; Economics, Pharmaceutical; Methodology (source: MeSH NLM).

\section{INTRODUCCIÓN}

En salud, el Análisis de Impacto Presupuestario (AIP) busca calcular los resultados financieros de adoptar y difundir una intervención en un programa de cobertura específica o sistema de salud. En particular, predicen cómo un cambio en las drogas $u$ otras terapias usadas para tratar una condición específica impactará en los costos ${ }^{(1)}$. En otras palabras, es la estimación financiera de los costos netos, que incorporará una institución sanitaria, al dar cobertura en una determinada intervención.

Los avances de los AIP en salud han llegado a plantear y reconocer que las Evaluaciones Económicas en Salud
(EES) analizan parte de las consecuencias de la incorporación de tecnologías sanitarias ${ }^{(2)}$. Las EES como los análisis de costo-efectividad o costo-utilidad estiman los costos y beneficios incrementales de una intervención en comparación a otra, pero no hablan de las implicancias financieras de tomar una decisión de cobertura. En este sentido, a pesar que un nuevo medicamento podría resultar en una alternativa costo-efectiva, también podría no ser financiable por una institución de cobertura. Los análisis de costo-efectividad o utilidad por sí solos no responden a las siguientes preguntas ¿puede el financiador dar cobertura a sus beneficiarios? ¿Se poseen recursos suficientes para afrontar la carga financiera de esta nueva tecnología?

Instituto de Efectividad Clínica y Sanitaria. Buenos Aires, Argentina

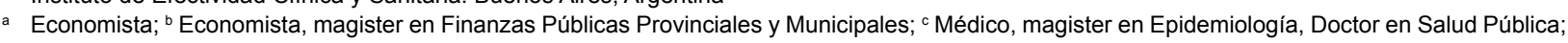

${ }^{\mathrm{d}}$ Médico de familia, magister en Efectividad Clínica; ${ }^{e}$ Médico de familia, Doctor en medicina

Recibido: 09-07-11 Aprobado: 14-09-11 
A pesar de la importancia de estas preguntas, los AIP no son vistos como EES o por lo menos aún se discute su consideración como tal (3) ya que con frecuencia se los asocia con ejercicios menos complejos. De esta forma, las guías actuales recomiendan desarrollar los AIP basándose en una evaluación económica ${ }^{(1,4,5)}$, debido a que ambos respetan la misma estructura epidemiológica. De todas maneras, es importante aclarar que es posible realizar estudios de impacto presupuestario de forma independiente, es decir prescindiendo de los análisis de costo-efectividad o utilidad.

En el mundo cada es vez mayor la necesidad de generar estudios de impacto presupuestario junto con análisis de costo-efectividad o utilidad ${ }^{(1,6)}$ como requisito para evaluar la asequibilidad de las tecnologías, aunque en la práctica solo unos pocos países las realizan, como Australia (2002), Bélgica (2002), Canadá (2003), Estados Unidos (2003), Francia (2005), Hungría (2002), Holanda (1999), Inglaterra (2004), Italia (2001), Israel (2004) y Polonia (2004) ${ }^{(2,3)}$.

En Latinoamérica son varios los avances en el campo de las EES, al punto que Brasil (2009) (7), Colombia $(2009){ }^{(8)}$, Cuba (2003) ${ }^{(9)}$ y México (2008) ${ }^{(10)}$ publicaron guías con recomendaciones locales. De ellos, solo Colombia considera a los estudios de impacto presupuestario como un componente importante de las evaluaciones económicas, mientras que los demás países le dan un carácter de opcional ${ }^{(8,11)}$. En las guías de México, por ejemplo, se señala como deseable la incorporación de este tipo de estudio pero no se realizan mayores comentarios $^{(10)}$ y en las guías de Cuba solo se establece que las conclusiones deben hacer referencia a la repercusión económica de los resultados ${ }^{(9)}$. En Brasil, si bien no existen fuertes recomendaciones en sus guías ${ }^{(7)}$, se han desarrollado políticas concretas para su desarrollo. En el año 2003 se creó, bajo la coordinación de la Agência Nacional de Vigilância Sanitária (ANVISA), la Unidad de Evaluación de Tecnologías Sanitarias (UETS), que debe, entre otras tareas, realizar evaluaciones de impacto presupuestario para asistir la inclusión de nuevas tecnologías al SUS. En Uruguay sucede algo similar con el Fondo Nacional de Recursos, que desde el 2007 plantea que ningún medicamento puede ingresar a sus listas antes de cumplir con ciertas condiciones entre las que se incluye un análisis de impacto presupuestario ${ }^{(12)}$.

Los objetivos de este artículo son: (i) identificar los determinantes y componentes de los análisis de impacto presupuestario y, a partir de ello (ii), describir el desarrollo de un modelo diseñado en una planilla de cálculo que permite considerar cualquier tecnología sanitaria y obtener estimaciones con diferentes grados de complejidad.

\section{EL MODELO Y SUS DETERMINANTES}

El modelo se desarrolla en Microsoft Excel y posee una estructura de fácil comprensión para promover su uso en personas con diferentes niveles de conocimiento en el campo de las evaluaciones de impacto económicosanitario. La Tabla 1 muestra la estructura general del modelo.

Tabla 1. Estructura del modelo, datos requeridos u observaciones según las principales secciones analíticas.

\begin{tabular}{|c|c|}
\hline Secciones del modelo & Datos requeridos / Observaciones \\
\hline \multicolumn{2}{|l|}{ Población } \\
\hline Cantidad de casos por año & Obs: Utilizando datos propios o ajustando datos de la literatura, encuestas o registros poblacionales \\
\hline \multicolumn{2}{|l|}{ Supuestos / Ajustes } \\
\hline Ajuste por indicación & Personas que efectivamente podrán recibir la tecnología \\
\hline Ajuste por adherencia & $\%$ de pacientes que efectivamente cumplen el tratamiento \\
\hline Ajuste temporal & Obs: tener en cuenta la diferencia entre enfermedades crónicas vs agudas \\
\hline Crecimiento poblacional & Obs: Incluir sólo si es muy importante \\
\hline Otros ajustes & Obs: Específicos de cada institución \\
\hline \multicolumn{2}{|l|}{ Costos } \\
\hline Costos de la nueva tecnología & Obs: Sólo considera los costos directos \\
\hline Drogas y dispositivos & Obs: Discriminar opciones de tratamiento \\
\hline Cobertura financiera & \% del costo de la tecnología que estará a cargo de la institución \\
\hline Costos asociados & Son las cargas financieras que posee la nueva tecnología además de su costo directo \\
\hline Componentes & Obs: Pueden ser drogas concomitantes, consultas, internaciones, etc. \\
\hline Efectos adversos & Se calcula de la misma manera que los componentes \\
\hline Diferenciales de costos & Son los ahorros o costos adicionales por reemplazar el tratamiento actual \\
\hline Reemplazo & Ahorros por reemplazo de tecnologías \\
\hline Variaciones & Variaciones por eficacia de la tecnología (pueden ser ahorros o costos adicionales) \\
\hline \multicolumn{2}{|l|}{ Supuestos / Ajustes } \\
\hline Inflación & Se recomienda incluir tasas diferenciales de acuerdo a su importancia en el modelo \\
\hline Tasa de difusión & $\%$ de pacientes que utilizan la tecnología en relación al comparador \\
\hline Horizonte temporal & Mediano plazo, de 1 a 5 años \\
\hline Perspectiva & Pagador \\
\hline Análisis de sensibilidad & "una vía" o multivariante \\
\hline Presentación & Costos esperados frente a una Visión probabilística \\
\hline
\end{tabular}




\section{POBLACIÓN}

Identificación de la población objetivo. En primer lugar, se debe identificar y delimitar la población objetivo de la enfermedad bajo estudio. En muchos casos las instituciones poseen registros confiables que permiten estimar los casos mediante simple extrapolación del pasado, de modo que ante esta situación, se debería calcular simplemente el promedio de los casos registrados por año. En cambio, en otros casos, esta información no se encuentra disponible debido a la inexistencia de registros, la sospecha de mala calidad de los datos o simplemente porque el análisis se basa en una tecnología sin un estándar con el cual compararlo. En esta situación, la estimación se basa en ajustes realizados con datos de la literatura. En primer lugar se debe estructurar la población general de acuerdo a la mayor cantidad de información posible, básicamente por sexo y edad, es decir, se debe armar un perfil epidemiológico de la institución afectada por el análisis. Luego se deben aplicar tasas de prevalencia o incidencia obtenidas de la literatura, las cuales deberían atravesar procesos de validación o ajustes que permitan la transferibilidad de los indicadores obtenidos. Frecuentemente, estos procesos implican métodos de consenso de expertos tales como paneles Delphi u otras técnicas similares.

\section{AJUSTES SOBRE LA POBLACIÓN OBJETIVO}

Adherencia a la tecnología. Este ajuste se asocia al comportamiento de las personas frente al hecho de cumplir con cierta indicación médica ${ }^{(13)}$ y trata de cuantificar en qué medida los tratamientos son seguidos adecuadamente por los pacientes a lo largo del periodo bajo análisis. La fuente de información habitual para este tipo de indicador es la literatura o en su defecto las consultas de expertos mediante distintos métodos de consenso.

Ajuste temporal, enfermedades crónicas frente a agudas. En términos financieros, para las enfermedades agudas, es razonable suponer que la cantidad de casos estimados por año serán iguales para todo el horizonte temporal; pero para las enfermedades crónicas esto puede variar anualmente. En el caso de las drogas oncológicas, por ejemplo, la sobrevida diferencial que podría aportar una nueva tecnología aumentaría o disminuiría el número de pacientes que recibirían el tratamiento. En este caso, la "prevalencia presupuestaria" aumentará al ritmo de la sobrevida que genere el medicamento (ceteris paribus) y afectará al presupuesto de forma creciente, mientras que en los casos que el tratamiento sea agudo, por ejemplo solo una vez en la vida, la cantidad de casos posibles a tratar disminuirá en caso de mayor efectividad (ceteris paribus) afectando al presupuesto de forma decreciente. De todas maneras, la importancia de este punto debe ser relativizada por parte del investigador teniendo en cuenta las posibilidades que posea de incorporarlas en su análisis.

Crecimiento de la población. Debido a que los cambios en la población objetivo pueden ser de vital importancia para los AIP, el modelo incorpora la posibilidad de realizar ajustes sobre este aspecto. Los motivos por los cuales la población podría cambiar en el tiempo son múltiples: (i) crecimiento poblacional natural; (ii) actividades de promoción generadas por las propias instituciones de salud; (iii) nuevos marcos regulatorios; entre otros factores. Independientemente de cuáles sean los motivos que generan el cambio en el número de casos a considerar, el factor escala puede ser importante dependiendo de las condiciones a evaluar.

Otros ajustes. Dado el carácter genérico del modelo se reconoce la posibilidad de realizar otros ajustes que podrían afectar a la población identificada, sin importar el método utilizado. Estos casos pueden responder, por ejemplo, a la cantidad de personas potencialmente incluibles dentro del grupo que puede afectar un presupuesto $\mathrm{u}$ otros casos como tasas de coberturas financieras en ciertas enfermedades, convenios con otras instituciones de salud, determinadas características que puedan afectar el acceso de los pacientes a las nuevas intervenciones o cualquier situación particular que deba ser considerada.

\section{ESTIMACIONES DE COSTOS}

La estimación del impacto en salud, es la identificación de los costos netos de una intervención, por lo que solo nos van a interesar los diferenciales financieros entre las tecnologías comparadas. La pregunta que responderá el investigador será sobre el impacto neto de la nueva tecnología. Podrá decir que en caso de incluir la tecnología dentro de su cartilla de prestaciones, habrá una diferencia con el costo de su actual tecnología y, en caso que no exista un tratamiento actual, el impacto neto será el costo global de la nueva tecnología.

Bajo esta lógica, la estructura de nuestro modelo se divide en tres secciones principales, la primera trata sobre la tecnología evaluada y las dos siguientes sobre los efectos diferenciales en relación a la tecnología a comparar. La Figura 1 muestra esta ecuación de costos.

Dado el carácter genérico de este modelo, un aspecto que debe ser mencionado es que cada uno de los puntos identificados en la ecuación puede ser estimado a través de un microcosteo de sus componentes o también de macrocostos, es decir, valores de paquetes 


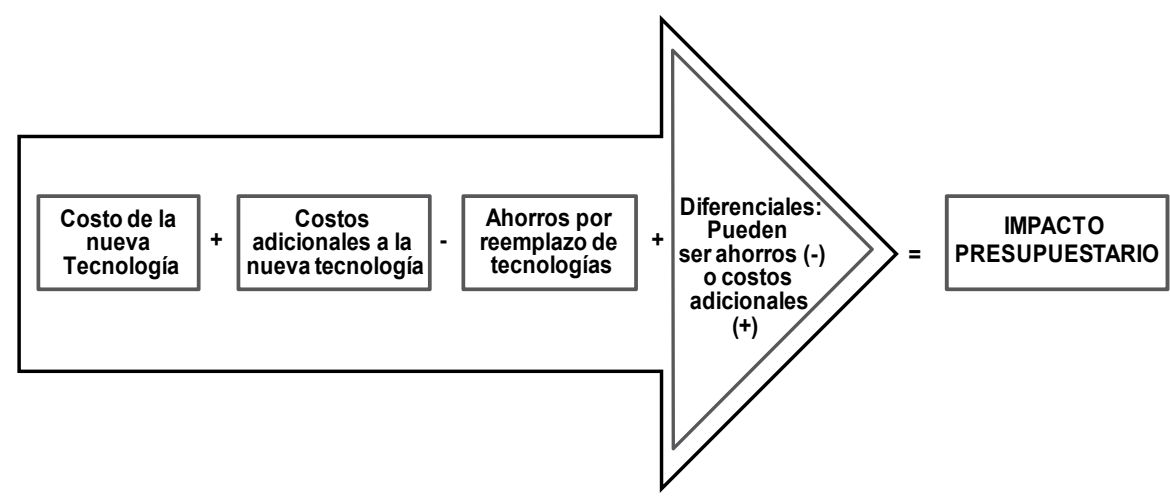

Figura 1. Cálculo del impacto presupuestario de acuerdo a las características de los costos y ahorros incluidos en el análisis.

de servicios o esquemas financieros que incluyen varias partidas de costos asociados.

\section{COSTOS DE LA NUEVA TECNOLOGÍA}

Dispositivos y drogas. Cuando la tecnología bajo estudio es un dispositivo, solo debe considerarse el costo de adquisición, pero en el caso que sea una droga, es necesario conocer ciertos parámetros asociados al tratamiento (cantidad de mg por unidad de tiempo) y a la presentación (mg por unidad, cantidad de unidades por presentación y precio) para la estimación del costo. A su vez, como los tratamientos pueden ser utilizados en forma distinta por diversas poblaciones (niños o adultos por ejemplo), en ocasiones puede ser necesario ajustar los resultados a determinados porcentajes de utilización. Por ejemplo, la dosificación puede variar de acuerdo con la edad y, si ésta se distribuyera en partes iguales entre niños y adultos, correspondería un $50 \%$ para cada población y tratamiento. Otro punto que puede ser determinante, y debe ser mencionado a la hora de estimar los costos de los tratamientos, son los porcentajes de "desperdicio" de las drogas. En el caso de las ampollas, se pueden perder varios miligramos por aplicación si la prescripción es menor a la presentación, aunque también es razonable suponer que en drogas muy costosas se reutiliza el restante de las ampollas para otras dosis. Si las pérdidas se suponen considerables, es necesario estimarlas con mayor precisión, dado que pueden tener un impacto importante en el resultado final. Nuestro modelo permite contemplar estos aspectos.

Componentes asociados al uso de nuevas tecnologías. Para estimar los costos netos debemos, en primer lugar, calcular los costos asociados al consumo o aplicación de nuevas tecnologías, los cuales pueden ser esquematizados a partir las siguientes categorías principales: a) Drogas concomitantes, donde se costea en forma similar al título anterior; b) Consultas médicas, donde se identifican costos unitarios por unidad de tiempo con la posibilidad de asociarlos a diferentes porcentajes de utilización; c) Costo de administración; d) Pruebas diagnósticas; e) Procedimientos; f) Otros costos. Todos estos valores deben sumarse al costo directo de la nueva tecnología.

Tal como se mencionó previamente, la estimación de los costos se basa en valores diferenciales, de forma tal que el modelo aquí propuesto solo contempla la inclusión de aquellos montos adicionales a los costos actuales. Este es un punto importante debido a que puede llevar a una sobreestimación de costos por lo que debe quedar muy claro. Por ejemplo, si el caso bajo análisis fuese el de una institución que está decidiendo la inclusión de una nueva droga en su formulario terapéutico, que obligaría al paciente a concurrir en vez de dos, tres veces por semana al hospital para supervisar su evolución, solo debería costearse una consulta médica por semana, no tres, ni dos, solo la consulta adicional al tratamiento anterior.

Cobertura. Las instituciones o sistemas de salud de salud poseen políticas diversas en cuanto a la cobertura de sus servicios, por lo que es necesario contemplar la posibilidad de realizar un ajuste directo sobre el costo de la nueva tecnología. En ciertos casos, podría suceder que se decida cubrir solo un porcentaje del costo de la nueva tecnología o se decida realizar una política de cobertura gradual. Estas situaciones pueden incluirse en este apartado.

Efectos Adversos. Estos costos pueden asociarse al consumo de nuevas drogas, consultas a especialistas, días de hospitalización, entre otros; y pueden manejarse bajo el mismo esquema detallado en el título de "Componentes asociados al uso de la nueva tecnología". En diversos casos los efectos adversos pueden ser significativos en términos de impacto presupuestario por lo que deben incluirse dentro de la estimación. 
Diferenciales de costos por el uso de la nueva tecnología. El cambio de una modalidad de tratamiento existente debido a la incorporación de una nueva tecnología puede derivar en efectos de sustitución de distintos recursos sanitarios ${ }^{(4)}$, es decir puede reemplazar tecnologías o modificar los procesos de producción vigentes de distintos tratamientos. En este sentido, es importante distinguir este efecto de sustitución, que solo genera ahorros, del de variación por eficacia, que puede generar ahorros o mayores costos financieros.

Un ejemplo de variación de eficacia asociada con un ahorro financiero podría ser el caso de un nuevo medicamento que disminuya la cantidad de consumo de otros medicamentos; por ejemplo, analgésicos con un mayor efecto para la disminución del dolor, por lo que el paciente debe acudir menos veces al médico. En este caso, el gasto sigue estando pero es menor. Bajo este esquema el pagador debe computar una menor cantidad de consultas médicas y gastos en analgésicos. En cambio, un ejemplo de reemplazo sería la simple sustitución de un medicamento o tratamiento viejo de menor eficacia, que no necesite tomar debido a las características del nuevo tratamiento.

Bajo este modelo se puede considerar el efecto de variación entre las siguientes categorías: variación en drogas que se siguen utilizando, modificación de la cantidad de consultas médicas, test diagnósticos, procedimientos y otros costos. El efecto sustitución se puede incluir en las siguientes categorías: dispositivos, drogas, consultas médicas, costos de administración, pruebas diagnósticas, procedimientos, otros costos y efectos adversos.

\section{HORIZONTE TEMPORAL}

El horizonte temporal debe ser lo suficientemente amplio como para incorporar todos los determinantes del modelo y debe estar basado en las necesidades del pagador ${ }^{(4,5)}$. En este sentido, el investigador podría optar entre los horizontes temporales más habituales, esto es entre un rango de dos a cinco años. Los períodos mayores a cinco años no son fáciles de abordar debido a la incertidumbre en los supuestos del modelo y por que son poco relevantes desde la óptica del pagador ${ }^{(2)}$.

\section{INFLACIÓN Y TASA DE DESCUENTO}

La variación de precios es un tema controvertido en los AIP ya que su inclusión o no, aún se encuentra bajo discusión. Las recomendaciones de las guías para la elaboración de análisis de impacto presupuestario en general tienden a no considerarla, pero aun así recomiendan que se brinde la posibilidad de incluirla utilizando análisis de distintos escenarios. Nuestro modelo permite hacerlo discriminando tasas por tipo de recursos, permitiendo también la asignación de un valor nulo a dichas tasas. En cuanto a la tasa de descuento, no es necesario considerarla, ya que los flujos financieros se comparan a lo largo del tiempo (1), además, desde la perspectiva de los costos diferenciales, al no comparar entre dos intervenciones sino solo el impacto bajo un comparador, la utilización del descuento como reflejo de oportunidades perdidas de inversión deja de tener valor.

\section{TASA DE DIFUSIÓN DE LA TECNOLOGÍA}

La difusión podemos definirla como la tasa progresiva de sustitución del tratamiento actual, respecto a la nueva tecnología ${ }^{(2)}$. Es razonable suponer que ante la decisión de un proveedor de salud de reconocer la tecnología en su cartilla de prestaciones, no todos los pacientes susceptibles de recibirla lo hagan al mismo tiempo, por lo que debemos estimar una evolución en la incorporación. Esta tasa puede estar determinada por diversos factores, desde lineamientos específicos de la institución o decisiones políticas, hasta características propias de la tecnología. El modelo permite incorporar distintas curvas de difusión para ser utilizadas con tecnologías de distinto nivel de difusión o estimaciones propias. De esta forma, se pueden reflejar los casos en los cuales la mayor parte de la población blanco va a acceder a la nueva tecnología apenas esté disponible o también en aquellos donde la tasa difusión es muy lenta, donde a pesar de estar disponible, la tecnología solo es incorporada gradualmente en el tiempo ${ }^{(4)}$.

\section{PERSPECTIVA DEL ANÁLISIS}

Debido a que los estudios de impacto presupuestario son análisis estrictamente financieros e interesan al decisor en la medida que puedan afectar sus presupuestos, la perspectiva debe ser la del pagador ${ }^{(1,4)}$. Si es una entidad pública, a la cual le interesan las implicancias económicas no solo de sus presupuestos institucionales sino también de la sociedad en general, se debería permitir la inclusión de pérdidas o ganancias secundarias de los individuos tales como pérdidas de oportunidad (por ejemplo, por ausentismo laboral) u otro tipo de gastos relevantes para el análisis, pero esto debería presentarse como un escenario adicional a la perspectiva del pagador.

\section{ANÁLISIS DE SENSIBILIDAD}

El objetivo del análisis de sensibilidad es describir cómo afectará cambiar los parámetros más inciertos y con mayor potencial de influencia en los resultados del estudio ${ }^{(3)}$. Respecto a las recomendaciones para su realización, existe un acuerdo entre las guías sobre la importancia de realizar análisis de este tipo ${ }^{(5)}$ 
fundamentado en la marcada presencia de incertidumbre en los AIP y la necesidad de estudiar su influencia en los resultados finales. El acercamiento convencional es de "una vía", donde se varía un parámetro a la vez y luego se observa el efecto de dicha variación en los resultados globales del modelo. Mientras que en un análisis de sensibilidad multivariable, más de un parámetro es modificado en forma simultánea corrigiéndose los efectos de cada uno sobre el resultado de interés ${ }^{(4)}$.

\section{PRESENTACIÓN DE LOS RESULTADOS}

Para la presentación de los resultados, es útil incorporar una visión probabilística de la ocurrencia de la enfermedad en análisis. Por ejemplo, algunas enfermedades "raras" poseen prevalencias muy bajas y generalmente los costos de sus tratamientos son muy elevados. De esta forma, si la institución que lleva adelante el estudio posee una población muy pequeña es probable que no se presente ningún caso por año, pero sus costos esperados podrían ser muy importantes. Por este motivo, es importante asociar la presentación de casos a probabilidades que permitan establecer rangos o criterios de decisión para definir los presupuestos.

Como ejemplo, a continuación se presentan dos casos hipotéticos para ejemplificar los resultados que ofrece el modelo. En el Caso 1 se estima el impacto presupuestario en una institución de salud importante en términos de afiliados y se considera un medicamento de bajo costo para una enfermedad de alta prevalencia. En este ejercicio se puede esperar un impacto financiero neto de USD 6250000 en el primer año que, de acuerdo a los diferentes supuestos, crece hasta USD 25000000 en el quinto, como se ve en la Figura 2.

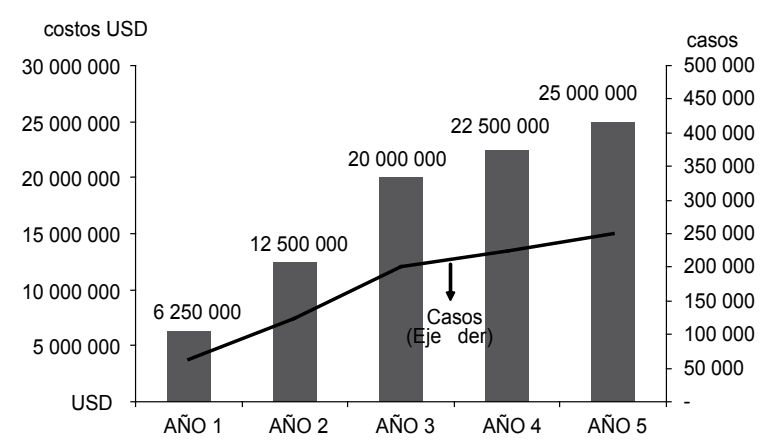

Figura 2. Ejemplo financiero del impacto presupuestario en una institución de salud importante.

\section{Datos}

Población de la institución:

5000000

Prevalencia: 5 / 1000 personas Impacto diferencial unitario (por tratamiento): USD 100

\section{Supuestos}

Tasa de difusión anual $=$ media (25\%; $50 \% ; 80 \% ; 90 \%$; $100 \%$ ) Crecimiento la población= Nulo Adherencia $=100 \%$ Inflación= $0 \%$ Cobertura financiera $=100 \%$
En el Caso 2 se presenta a una institución pequeña con un medicamento de alto costo para tratar una enfermedad de baja prevalencia. En esta oportunidad, se observa en términos financieros un impacto esperado de USD 312500 en el primer año y USD 1519383 en el quinto, mientras que los casos esperados oscilan entre 0 y 3 durante todo el horizonte temporal, como se ve en la Figura 3.

\section{DISCUSIÓN}

El modelo de impacto presupuestario presentado en este artículo se diseñó para incluir los componentes y supuestos considerados relevantes y, además, se estructuró a partir de la consideración de las capacidades de los usuarios. La idea subyacente bajo esta estructura es la utilización directa por quienes necesitan las estimaciones, es

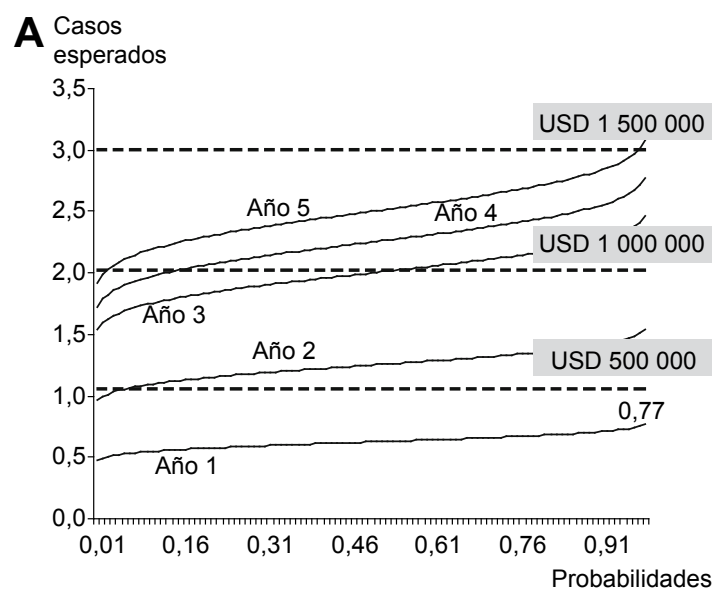

B

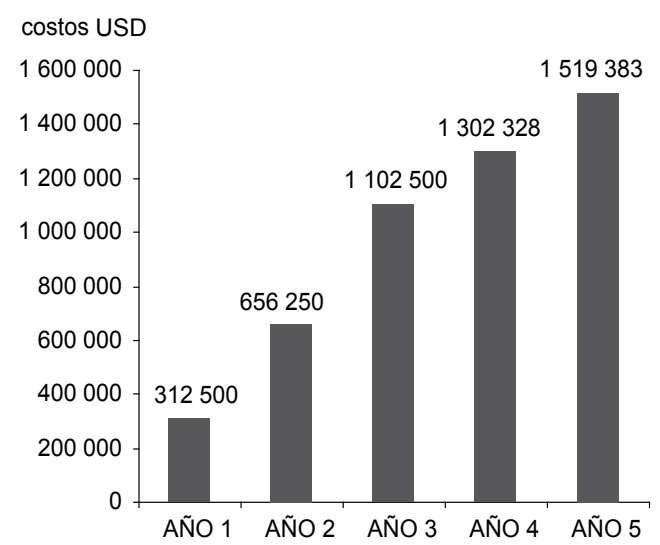

Figura 3. Ejemplo financiero en A y B del impacto presupuestario en una institución de salud pequeña.
Datos

Población de la institución: 50000 Prevalencia: 5 / 100000 personas Impacto diferencial unitario (por tratamiento): USD 500000

\section{Supuestos}

Tasa de difusión anual= media (25\%; $50 \% ; 80 \% ; 90 \% ; 100 \%)$ Crecimiento de la población= alto Adherencia $=100 \%$ Inflación= $0 \%$

Cobertura financiera $=100 \%$ 
decir, los propios decisores en salud. En general, este público no está familiarizado con los métodos para desarrollar EES y AIP, de modo que el diseño propuesto pretende tomar esto en cuenta y conseguir, al mismo tiempo, aumentar la transparencia de los resultados, un punto muy mencionado en los trabajos metodológicos ${ }^{(1-5)}$. Este tema lo consideramos un aspecto clave para la estrategia de difusión y desarrollo gradual de los análisis en los ámbitos de gestión de nuestros países, donde la toma de decisión es más rápida que los estudios que puedan hacerse. La promoción de la toma de decisiones basada en argumentos académicos debe respetar los tiempos, habilidades y recursos de los decisores y, si bien se desea que vayan juntas, no se deben entorpecer. Por este motivo, el modelo se diseñó de un modo flexible a diferentes niveles de complejidad, y así permitir "escalar" los requerimientos de información y realizar las estimaciones con diferentes grados de complejidad y disponibilidad.

Bajo esta perspectiva es muy importante señalar las limitaciones este abordaje, las cuales se encuadran dentro de la metodología consensuada ${ }^{(1-5)}$. En este sentido, creemos que el punto de mayor debilidad es el tratamiento de la incertidumbre. El carácter de los supuestos discutidos aquí los hace muchas veces difíciles de abordar, con fuentes de información muy escasas e incluso en ciertos casos inexistentes. Nuijten et al concluyen que gran parte de la información necesaria solo puede ser derivada a través de paneles Delphi ${ }^{(4)}$ lo cual es una dificultad ya que en muchos casos, por diferentes motivos, no es posible llevarlos a cabo. Nuestra recomendación es que en los casos que no puedan hacerlos se basen en sus propios datos o escenarios relevantes. Esta limitación en la información no debería significar una barrera infranqueable para el desarrollo de estos estudios ya que el objetivo del AIP, bajo el esquema propuesto, es ayudar a la toma de decisión y no conformarse como un método para reemplazarla ${ }^{(14)}$. Debería servir para reunir la mejor información disponible y realizar estimaciones que sirvan al momento de decisión; en este sentido creemos que poseer resultados aproximados, propios, bajo consideración de los supuestos realizados representa un escenario más adecuado que uno en donde no haya ningún dato al respecto.

Otro punto en el que se debe poner especial énfasis es el problema asociado a los supuestos que se incorporan al modelar la realidad y que no escapan a este modelo. En particular, un obstáculo importante para los AIP es cómo predecir si los ahorros son realmente realizables en la práctica ${ }^{(2)}$. En muchos casos, debido a la estructura de contratación de profesionales o pagos por servicios no es posible identificar fácilmente los diferenciales de costos asociados a la inclusión de nuevas tecnologías debido a que dichas variaciones no se traducen direc- tamente a dinero, por ejemplo, reducciones de tiempos de trabajo o cambios en los manejos de stocks o capitas pueden no efectivizarse en términos monetarios. Esta situación debe estudiarse con detenimiento para evitar sobre o subestimaciones en los resultados de impacto.

\section{CONCLUSIÓN}

Cada vez se vuelve más importante realizar AIP como complementos de las evaluaciones económicas en salud $^{(1,3-5,7)}$ pero no existe un consenso sobre los métodos para llevarlos a cabo ${ }^{(3,5)}$. En este artículo presentamos un modelo que busca incorporar en forma explícita las habilidades del usuario y las deficiencias de información, buscando promover el desarrollo de AIP en los ámbitos de gestión de nuestros países. Este modelo no escapa a las limitaciones generales que se presentan hoy en día pero aún así su uso no debería estar condicionado por esto. En ese sentido, concordamos con las palabras de Juarez-Castello "Pretender establecer una técnica que dé soluciones irrefutables es imposible, pero la alternativa no puede ser que las decisiones no se fundamenten en análisis de racionalidad económica y queden a la discrecionalidad o al capricho del decisor, o en manos de la capacidad de presión de la industria correspondiente" (14).

\section{Contribuciones de autoría}

Todos los autores participaron de la concepción, diseño, y redacción del manuscrito; todos aprueban la publicación de la versión final.

\section{Conflictos de interés}

Los autores no presentan ningún conflicto de interés con los temas discutidos en el manuscrito.

\section{Fuente de financiamiento}

El artículo ha sido financiado por el Instituto de Efectividad Clínica y Sanitaria. Buenos Aires, Argentina.

\section{REFERENCIAS BIBLIOGRÁFICAS}

1. Mauskopf JA, Sullivan SD, Annemans L, Caro J, Mullins CD, Nuijten M, et al. Principles of good practice for budget impact analysis: report of the ISPOR Task Force on good research practices--budget impact analysis. Value Health. 2007;10(5):336-47.

2. Trueman P, Drummond M, Hutton J. Developing guidance for budget impact analysis. Pharmacoeconomics. 2001;19(6):609-21

3. Brosa M, Gisbert R, Rodríguez JM, Soto J. Principios, métodos y aplicaciones del análisis del impacto presupuestario en el sector sanitario. Pharmacoeconomics - Spanish Research Articles. 2005;2(2):65-78. 
4. Nuijten MJ, Mittendorf T, Persson U. Practical issues in handling data input and uncertainty in a budget impact analysis. Eur J Health Econ. 2011;12(3):231-41.

5. Mauskopf JA, Earnshaw S, Mullins CD. Budget impact analysis: review of the state of the art. Expert Rev Pharmacoecon Outcomes Res. 2005;5(1):65-79.

6. Orlewska E, Mierzejewski P. Proposal of Polish guidelines for conducting financial analysis and their comparison to existing guidance on budget impact in other countries. Value Health. 2004;7(1):1-10.

7. Ministério da Saúde de Brasil. Diretrizes metodológicas para elaboração de pareceres técnico-científicos para o Ministério da Saúde. Brasilia: Ministério da Saúde de Brasil; 2007.

8. Ministerio de la Protección Social de Colombia. Guía metodológica para la elaboración de Guías de Práctica Clínica basadas en la evidencia, de evaluaciones económicas y de evaluación del impacto de la implementación de las guías en el POS y en la Unidad de Pago por Capitación del Sistema General de Seguridad Social en Salud Colombiano. Bogotá: Ministerio de la Protección Social, COLCIENCIAS; 2008.

9. Galvez González AM. Guía metodológica para la evaluación económica en salud. Cuba, 2003. La Habana: Ministerio de Salud Pública de Cuba; 2004.
10. Consejo de Salubridad General de México. Guía para la conducción de estudios de evaluación económica para la actualización del Cuadro Básico de Insumos del Sector Salud en México. México: Consejo de Salubridad General; 2008.

11. Augustovski F, Garay OU, Pichon-Riviere A, Rubinstein A, Caporale JE. Economic evaluation guidelines in Latin America: a current snapshot. Expert Rev Pharmacoecon Outcomes Res. 2010;10(5):525-37.

12. Ministerio de Economía y Finanzas, Ministerio de Salud Pública. Decreto No 265/006. Montevideo: República Oriental de Uruguay; 2006. (7 de agosto de 2006).

13. Sabate E, De Geest S. Adherence to long-term therapies management: a call for cardiovascular nursing managers and policymakers. Prog Cardiovasc Nurs. 2004;19(1):28-9.

14. Juárez-Castelló $\mathbf{C}$, Rodríguez-Ibeas $\mathbf{R}$. Coste-efectividad e impacto presupuestario en la toma de decisiones de introducción de nuevas tecnologías: comentarios al artículo de Pinto et al. Gac Sanit. 2008;22(6):585-95.

Correspondencia: Osvaldo Ulises Garay

Dirección: Dr. Emilio Ravignani 2024, Buenos Aires, Argentina.

Teléfono: (+54-11) 4777-8767

Correo electrónico: ugaray@iecs.org.ar

\section{Consulte la versión electrónica de la Revista Peruana de Medicina Experimental y Salud Pública en}

\section{WWW.scopus.com}

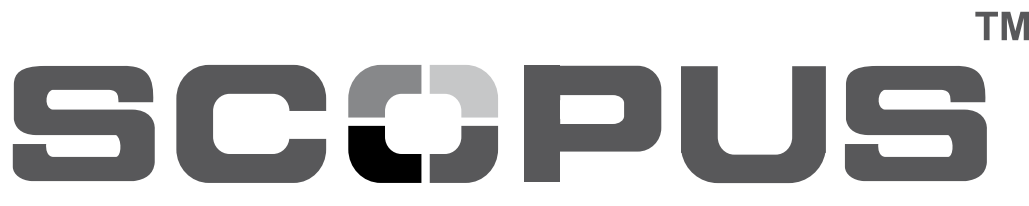

